\title{
SGCE isoform characterization and expression in human brain: implications for myoclonus-dystonia pathogenesis?
}

\author{
Katja Ritz ${ }^{1,4}$, Barbera DC van Schaik ${ }^{2}$, Marja E Jakobs ${ }^{1}$, Antoine H van Kampen ${ }^{2,5}$, Eleonora Aronica ${ }^{3}$, \\ Marina A Tijssen ${ }^{4}$ and Frank Baas ${ }^{\star, 1}$ \\ Myoclonus-dystonia (M-D) is a neurological movement disorder with involuntary jerky and dystonic movements as major \\ symptoms. About $50 \%$ of M-D patients have a mutation in $\varepsilon$-sarcoglycan (SGCE), a maternally imprinted gene that is widely \\ expressed. As little is known about SGCE function, one can only speculate about the pathomechanisms of the exclusively \\ neurological phenotype in M-D. We characterized different SGCE isoforms in the human brain using ultra-deep sequencing. \\ We show that a major brain-specific isoform is differentially expressed in the human brain with a notably high expression in the \\ cerebellum, namely in the Purkinje cells and neurons of the dentate nucleus. Its expression was low in the globus pallidus and \\ moderate to low in caudate nucleus, putamen and substantia nigra. Our data are compatible with a model in which dysfunction \\ of the cerebellum is involved in the pathogenesis of M-D.
}

European Journal of Human Genetics (2011) 19, 438-444; doi:10.1038/ejhg.2010.206; published online 15 December 2010

Keywords: myoclonus-dystonia; SGCE; deep sequencing; alternative splicing; imprinting; cerebellum

\section{INTRODUCTION}

Myoclonus-dystonia (M-D) is a movement disorder characterized by myoclonic jerks and dystonic features, usually affecting the upper part of the body. Little is known about the pathological basis of $\mathrm{M}-\mathrm{D}$ symptoms. Electrophysiological studies in man suggest that myoclonic symptoms are of subcortical origin. ${ }^{1-3}$ In general, dystonia is thought to arise from dysfunction of the basal ganglia. ${ }^{4}$ About $50 \%$ of $\mathrm{M}-\mathrm{D}$ patients who were classified as definite $\mathrm{M}-\mathrm{D}$ carry a mutation in the widely expressed $\varepsilon$-sarcoglycan $(S G C E) .^{5-7}$ The genetic cause in the remaining patients is still unclear. A second locus has been reported in one large M-D family (DYT15, 18p11), but no gene has been identified yet. ${ }^{8,9}$ SGCE is part of the sarcoglycan family that consists of $\mathrm{N}$-glycosylated transmembrane proteins. Six different sarcoglycans have been identified so far $\left(\alpha-, \beta-, \gamma-, \delta-, \varepsilon_{-}\right.$and $\left.\zeta_{-}\right)$, but little is known about the function of particular sarcoglycan members and their function in different tissues. ${ }^{10}$ In muscles, sarcoglycans form a heterotetrameric complex that is constituent of the dystrophinassociated protein complex. This complex mediates the structural stability of the plasma membrane and interactions between the extracellular matrix and the cytoskeleton. ${ }^{11}$ Mutations in other sarcoglycans, $\alpha-, \beta$-, $\gamma$ - and $\delta$-sarcoglycan, lead to different forms of limb-girdle muscular dystrophy, characterized by progressive muscle weakness. ${ }^{10}$ Little is known about composition and function of the dystrophin-associated protein complex in the brain. SGCE is highly homologous to $\alpha$-sarcoglycan, ${ }^{12}$ but no muscle or myocardial muscle abnormalities have been identified in $\mathrm{M}-\mathrm{D}$ patients. $^{13}$

Understanding the exclusively neurological phenotype of the disease is still a major challenge. SGCE is an imprinted gene, ${ }^{14,15}$ meaning that a loss of function mutation in the expressed allele will be dominant. All reported mutations behave as null alleles, as they result in premature stop codons and are predicted to lead to nonsensemediated decay. There is also evidence that SGCE missense mutations lead to the loss of function. ${ }^{16} S G C E$ is widely expressed, which suggests either redundancy for this protein in non-brain tissue or a brainspecific function for SGCE.

There are four known alternatively spliced exons in the SGCE gene (exons 2, 8, 10 and 11b; Figure 1a), with exon 11b as a brain-specific exon. ${ }^{17}$ Recently, a new brain-specific alternatively spliced exon (exon 11c, an elongated exon 11b) has been identified in mice. ${ }^{18}$ Transcripts containing either exon $11 \mathrm{~b}$ or $11 \mathrm{c}$ encode proteins with a different C-terminal sequence containing a PDZ-binding motif. This motif is a protein-interaction domain and thus may contribute to a unique SGCE function in the brain. The ubiquitous SGCE protein and the brain-specific protein (exon 11b) are localized in different synaptosomal membrane fractions: post- and pre-synaptic membrane, respectively. ${ }^{17}$ It has been speculated that both isoforms play different roles at neuronal synapses.

The aim of our study was to characterize the quantitative and qualitative expression pattern of SGCE isoforms in the human brain to identify brain regions associated with $\mathrm{M}-\mathrm{D}$.

\footnotetext{
${ }^{1}$ Department of Genome Analysis, Academic Medical Center, University of Amsterdam, Amsterdam, The Netherlands; ${ }^{2}$ Bioinformatics Laboratory, Academic Medical Center, University of Amsterdam, Amsterdam, The Netherlands; ${ }^{3}$ Department of (Neuro)Pathology, Academic Medical Center, University of Amsterdam, Amsterdam, The Netherlands; ${ }^{4}$ Department of Neurology, Academic Medical Center, University of Amsterdam, Amsterdam, The Netherlands; ${ }^{5}$ Biosystems Data Analysis, Swammerdam Institute for Life Science, University of Amsterdam, Amsterdam, The Netherlands

*Correspondence: Dr F Baas, Department of Genome Analysis, Academic Medical Center, University of Amsterdam, Meibergdreef 9, Amsterdam 1105AZ, The Netherlands. Tel: +31 20566 3846; Fax +31 20566 9312; E-mail: f.baas@amc.uva.nl

Received 2 August 2010; revised 20 October 2010; accepted 21 October 2010; published online 15 December 2010
} 


\section{METHODS}

Sample collection and preparation

Human tissue was obtained from six control subjects without neurological symptoms (Table 1). In addition, two blood samples were obtained from control subjects. SGCE mutations were excluded by direct sequencing of the coding region. Human tissues were obtained from the Department of Neuropathology of the Academic Medical Center (University of Amsterdam, Amsterdam, the Netherlands) and informed consent was obtained for research purposes in all control subjects. Mouse (cerebral cortex), rat (cerebral cortex) and zebrafish (100 embryos, $24 \mathrm{~h}$ after fertilization) tissue was obtained from wild-type animals (approved by local committee). DNA extractions from frozen brain tissues and whole-blood samples were performed using standard procedures. Sections (20-40, depending on tissue size, $20 \mu \mathrm{m})$ were cut per sample and dissolved in TRIZOL Reagent (Invitrogen, Breda, the Netherlands) for subsequent RNA isolation, or in SE buffer (750 mM NaCl (pH 8), 250 mм EDTA, 1\% SDS) containing Proteinase K for DNA isolation. Total RNA was isolated with QIAcube instrument (Qiagen, Venlo, the Netherlands) using RNeasy Mini protocol or using the PAXgene system (Qiagen) for blood samples.

\section{Methylation-sensitive high-resolution melting assay}

To determine the degree of methylation in the promoter region of SGCE, we applied a methylation-sensitive high-resolution melting (MS-HRM) assay. The region amplified (nucleotide position -1148 to -773 relative to the start ATG) contains $25 \mathrm{CpG}$ sites and has been shown to be differentially methylated. ${ }^{14}$ One microgram of genomic DNA was chemically modified with sodium bisulfite using the EZ Methylation kit (Zymo Research, Orange, CA, USA) and amplified in the presence of ResoLight HRM dye (Roche Diagnostics, Almere, the Netherlands). Both methylated and unmethylated strands were amplified..$^{14}$ MS-HRM analysis was performed with the LightCycler 480 (Roche Diagnostics) and analyzed with LightCycler 480 gene scanning software module. In each run a set of standards of the identical SGCE promoter region was included (100, 50, 25, 12.5, 6.25 and $0 \%$ methylated). Different brain regions of five control subjects (subjects 1, 2, 4, 5 and 6), muscle tissue of two control subjects (subjects 2 and 3 ) and blood of 12 definite M-D SGCE nonmutation carriers ${ }^{7}$ were tested. A subset of PCR-amplified samples was cloned (pGEM-T Easy vector; Promega, Leiden, the Netherlands) and sequenced to verify results of the methylation assay (ABI big dye v3.1 chemistry, ABI 3730 sequencer; Applied Biosystems, Foster City, CA, USA).

\section{Ultra-deep amplicon sequencing}

cDNA was synthesized from $1 \mu \mathrm{g}$ of total RNA with oligod $\mathrm{T}_{12}$-VN primers and $1 \mu \mathrm{l}$ cDNA was subjected to a $10-\mu \mathrm{l}$ PCR reaction. PCR reactions were performed using fusion primers consisting of a 19-bp fixed sequence (Roche/ 454 GS FLX, A or B sequence at the $5^{\prime}$ end) and a target-specific sequence ( $3^{\prime}$ end). We added a 5-nucleotide multiplex identifier-tag to allow for multiplexing of samples (primer sequences and PCR conditions are available on request). Amplicons were processed and ultra-deep sequenced using the 454 GS FLX system (Roche Diagnostics) according to manufacturer's instructions.

\section{Data analysis}

To identify all alternatively spliced events, sequences were first grouped by a multiplex identifier-tag. Second, we used the BLAT algorithm (BLAST-like alignment tool) to compare each sequence with all other sequences to identify similar exon combinations per sample. Sequences were grouped when they encompassed at least 210 nucleotides, had a percent identity of at least $98 \%$, a score above 105 and a query coverage above $98 \%$. The resulting groups were mapped to the respective chromosome (BLAT algorithm, hg18 build) and analyzed in the UCSC genome browser and using CodonCode Aligner software 3.0.1 (Dedham, MA, USA).

\section{Real-time quantitative PCR}

cDNA $(1 \mu \mathrm{l})$ was amplified in triplicates using the LightCycler 480 (Roche Diagnostics) in a final volume of $10 \mu \mathrm{l}$ containing SYBR Green I Master mix (Roche Diagnostics). Intron spanning primers were designed to target either all $S G C E$ isoforms or the brain-specific isoform containing exon 11b. All quantitative PCR (qPCR) results were normalized against the housekeeping genes GAPDH and eEF-1 alpha. Data were analyzed using LinRegPCR analysis program. ${ }^{19}$

\section{In situ hybridization}

In situ hybridization (ISH) was performed as described previously. ${ }^{20}$ Two $5^{\prime}$-fluorescein-labeled 19mer antisense oligonucleotides containing locked

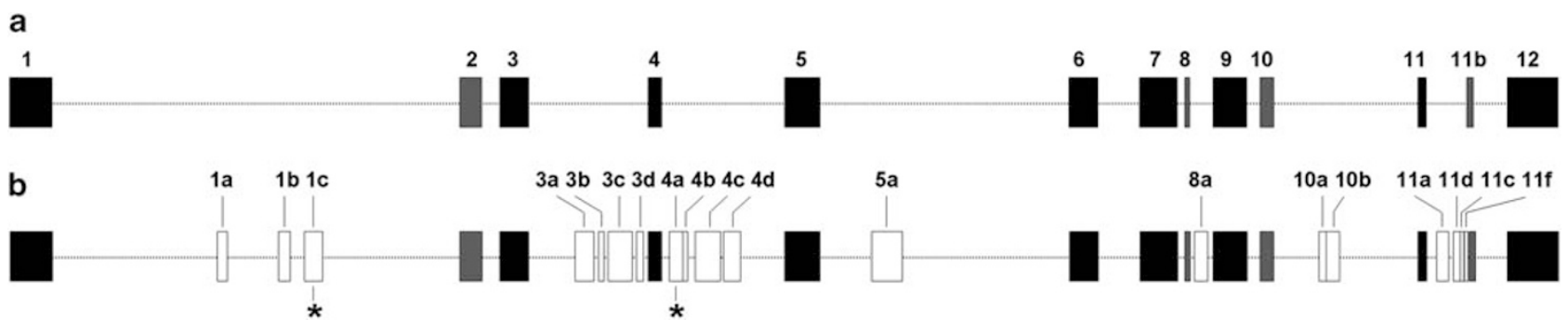

Figure 1 Overview of novel alternatively spliced exons identified by 454 ultra-deep sequencing. (a) SGCE gene including all known exons. Constitutive exons are depicted in black, and alternatively spliced exons in gray. (b) SGCE gene including novel alternatively spliced exons (in white). *Represent novel in-frame exons. In-frame exons were observed with a higher frequency (range: $0.3-5.7 \%$ ) than frameshift exons (range: $0.01-3 \%$ ). Relative position of the exons and sizes of exons are correct, but exon sizes are not represented in the same scale as the introns.

Table 1 Sample material

\begin{tabular}{llcll}
\hline Subject & Gender & Age (years) & Post mortem $(h)$ & Brain tissue \\
\hline 1 & Male & 77 & 7 & Other \\
2 & Male & 76 & 8 & Cb GP Hi M1 NC Pu SM1 SN Th \\
3 & Female & 76 & 5 & Cb GP Hi M1 NC Pu SM1 SN Th MP \\
4 & Male & 74 & 5 & NA \\
5 & Female & 88 & Cb GP Hi M1 NC Pu SM1 SN Th \\
6 & Female & 45 & Cb GP Hi M1 NC Pu SM1 SN Th & He MP \\
\hline
\end{tabular}

Abbreviations: $\mathrm{Cb}$, cerebellum; GP, globus pallidus; Hi, hippocampus; M1, primary motor cortex; NC, caudate nucleus; Pu, putamen; SM1, primary somatosensory cortex; SN, substantia nigra; Th, thalamus; He, heart; MP, m. psoas; NA, not available. 
nucleic acid and 2'-O-methyl RNA moieties (Ribotask ApS, Odense, Denmark) were designed: one targeting the brain-specific alternatively spliced exon $11 \mathrm{~b}$ (5'-AacGaaAauCucCugTagT- ${ }^{\prime}$, locked nucleic acid residues are given in capital letters, and 2'-O-methyl-RNA in lower case) and one detecting all isoforms targeting the constitutive SGCE exon 3 (5'-TagGacTccAucAcuAuaT-3'). Brain tissue was treated with Proteinase $\mathrm{K}\left(200 \mu \mathrm{g} / \mathrm{ml}\right.$; Roche Diagnostics) at $50^{\circ} \mathrm{C}$ for $10 \mathrm{~min}$. Probe annealing was performed at $53^{\circ} \mathrm{C}$ for $1.5 \mathrm{~h}$. Peroxidase activity was visualized using NovaRED (Vectorlabs, Burlingame, CA, USA) and counterstained with hematoxylin. In an adult control subject, we tested different human brain regions (cerebellum, mesencephalon, caudate nucleus, putamen, globus pallidus, cerebral cortex and hippocampus). Skeletal muscle, heart, kidney and liver sections were used to confirm specificity of the brainspecific probe. As a control for specific binding, brain material of an SGCE mutation carrier (c.835_839del, p.Lys280SerfsX16, 50-h post mortem) was analyzed. This tissue was obtained from the NICHD Brain and Tissue Bank (Baltimore, MD, USA).

\section{RESULTS}

\section{SGCE imprinting and $\mathrm{M}-\mathrm{D}$}

SGCE is widely expressed and there is thus far no explanation for the brain-specific phenotype of SGCE mutation carriers. One explanation could be that brain regions, which are not affected, are rescued by expression of the maternally imprinted allele. Therefore, we analyzed the SGCE imprinting throughout the human brain with an MS-HRM assay. We observed that the imprinting pattern was maintained in all brain regions and tissues tested (Supplementary Figure 1). Results of the MS-HRM assay were confirmed by cloning and direct sequencing of a subset of samples. Only minor variations in the number of methylated/unmethylated CpGs were detected by cloning, but not by the MS-HRM assay.

\section{SGCE mRNA expression by ultra-deep amplicon sequencing}

Different $S G C E$ isoforms have been reported so far, but little is known about their distribution and expression levels throughout the brain. To qualitatively and quantitatively characterize alternatively spliced $S G C E$ exons, we performed a systematic analysis of SGCE cDNA by ultradeep amplicon sequencing. This technique provides a powerful and accurate tool to investigate relative mRNA expression levels and detects rare and unknown splice events. To cover all exon-exon boundaries, four overlapping SGCE amplicons were made (exons $1-5,4-6,5-9$ and 7-12) and sequenced with a 454 GS FLX sequencer. Ultra-deep sequencing was achieved by aiming for 10000 sequence reads per amplicon (average $( \pm S D)$ : 11107 ( \pm 4273 ) reads; Supplementary Table 1).

SGCE mRNA isoforms. To get an overview of all alternative splicing events and their tissue specificity, we analyzed alternative splicing events of the entire SGCE gene in one control subject (subject 1) in the primary somatosensory cortex (SM1), heart and blood. Twenty-three different splicing events of the SGCE gene were detected, but only four of them occurred at frequencies above 1\%: exon 1c and the known alternatively spliced exons 2,8 and $11 \mathrm{~b}$ (Figure $1 \mathrm{~b}$, Table 2 ). In-frame exon 1c was expressed not only in the brain (5.7\%), but also in

Table 2 Known and new alternatively spliced SGCE exons revealed by screening the entire SGCE gene in one control subject with ultra-deep sequencing

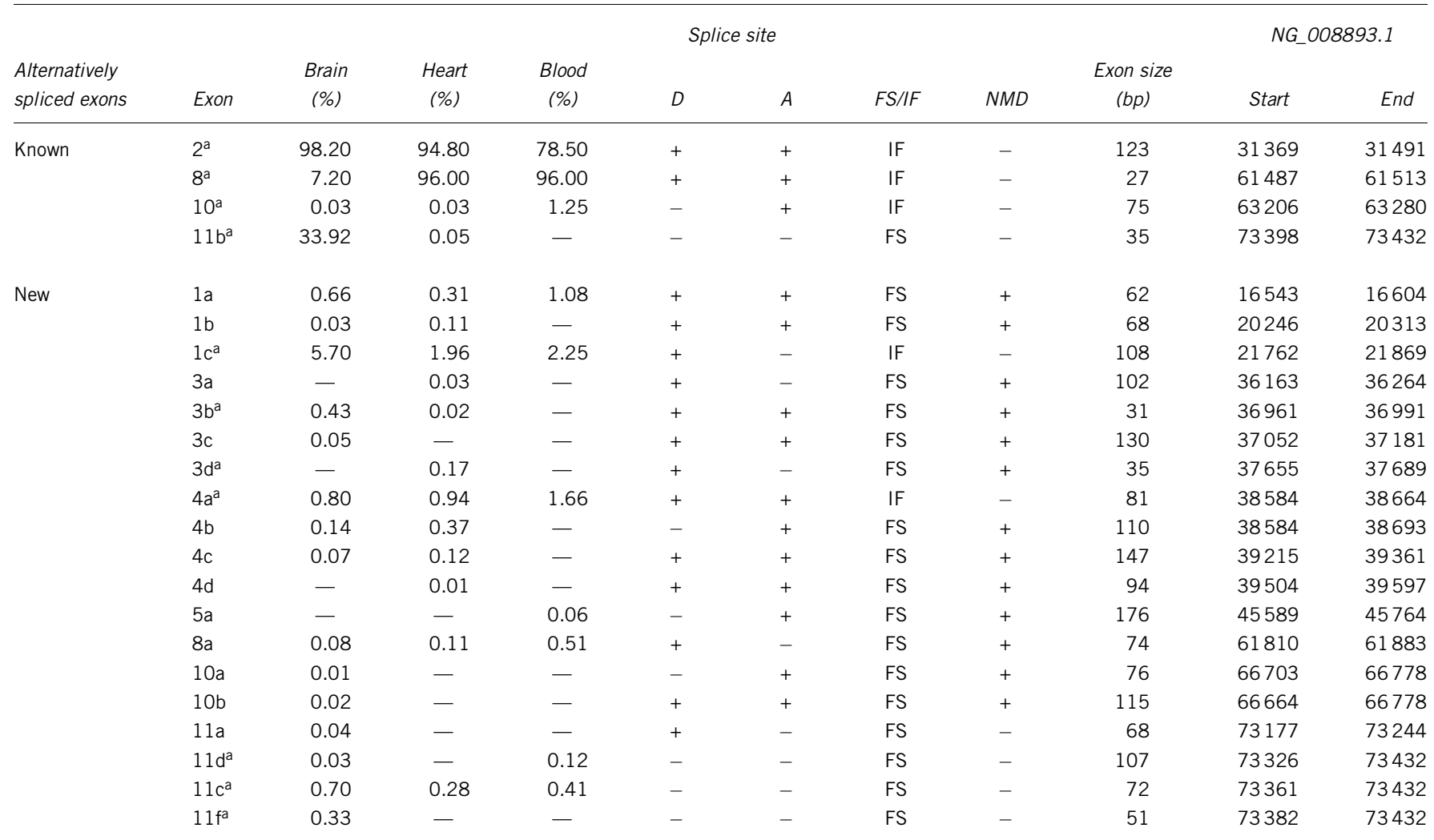

Additional information of alternatively spliced SGCE exons include: occurrence of respective exons in the brain (SM1), heart and blood of control subject 1 in percent; splice sites according to www.fruitfly.org, NNSPLICE 0.9 version (minimum score for $3^{\prime} / 5^{\prime}$, splice site 0.5 ): splice donor (D)/acceptor (A), frameshift (FS) or in-frame (IF) exons, isoform subject to nonsense-mediated decay www.fruitfly.org, NNSPLICE 0.9 version (minimum score for $3^{\prime} / 5^{\prime}$, splice site 0.5): splice donor (D)/acceptor (A), frameshift (FS)
(NMD), exon size in bp, nucleotide position according to NG_008893.1. Constitutive exons (100\% expression) are not shown.

aExons that were sequenced in definite M-D patients, in which no mutation could be identified. 
non-brain tissue $(2-2.3 \%)$. The in-frame exon 2 showed an overall high inclusion level in all tissues tested, whereas exon 8 was highly represented in muscle and blood and low in the brain. Exon 11b was mainly expressed in the brain (34\%); it was not expressed in blood and at very low levels in muscle $(0.05 \%)$. These four major variants result in a partially altered protein coding region and are extended into the last exon. Therefore, we expect these to be translated and stable. Of the low-frequency variants, only one, exon $4 \mathrm{a}$, retains a long open reading frame. All other mRNA isoforms, except all $11 \mathrm{~b}$ variants, are expected to result in nonsense-mediated decay. ${ }^{21}$ Splice site predictions and positions of known and novel exons are listed in Table 2. Most of the new low frequent variants lead to a frameshift $(17 / 19)$, resulting in a premature stop codon and were conserved among primate lineage only (Table 2, Supplementary Table 2). All known constitutive SGCE exons were present to $100 \%$, except for exon 11: skipping of this exon was observed in all tissues and species tested, leading to a premature stop after amino acid 409, albeit at low frequency (brain: $0.8 \%$; muscle: $1.4 \%$; blood: $0.3 \%$ ). The recently identified brain-specific exon $11 \mathrm{c}$ was expressed at very low levels not only in the brain, but also non-brain tissue $(\leq 0.7 \%$; Table 2$)$. Also, in mouse and rat brain it was expressed at very low levels $(<2.1 \%)$, and it was not expressed in zebrafish.

To exclude that these low-frequency variants were experimental artifacts, we sequenced a synthetic SGCE RNA using the same procedure. Analysis of 12000 reads gave only sequence reads identical to the input RNA (data not shown).

Brain region-specific SGCE expression. SGCE exon $11 \mathrm{~b}$ was the most abundant and a highly conserved brain-specific splice variant. Therefore, we analyzed exon $11 \mathrm{~b}$ expression in more detail and tested nine different human brain regions of two control subjects and three different organisms (mouse and rat brain, zebrafish) by ultra-deep amplicon sequencing.

Exon $11 \mathrm{~b}$ showed differential expression among the different brain regions in both control subjects. Levels were highest in SM1 and motor cortex (M1), low in the globus pallidus, intermediate in caudate nucleus and substantia nigra, and interindividual variation was found in the cerebellum and putamen (Table 3). In mouse and rat brain, we observed a higher rate of exon $11 \mathrm{~b}$ inclusion compared with the human brain (SM1: 50\% in mouse; $73 \%$ in rat $v s 35 \%$ in human). In whole zebrafish RNA, exon $11 \mathrm{~b}$ was present in $20 \%$ of transcripts.

\section{SGCE mRNA expression by qPCR}

Ultra-deep amplicon sequencing revealed that exon $11 \mathrm{~b}$ is the major brain-specific alternatively spliced SGCE exon and that it is differentially expressed in the human brain. Similar trends in expression levels of exon $11 \mathrm{~b}$ among the different brain regions were identified, and we also observed interindividual variability for some regions. To further characterize the expression pattern of SGCE transcripts, we tested the same brain regions of more control subjects by qPCR (Figure 2). We found similar trends of exon $11 \mathrm{~b}$ expression in five control subjects: expression in the cerebellum was significantly higher compared with globus pallidus (Figure 2; $P<0.001$, Friedman's test, Dunn's multiple comparison test) and substantia nigra $(P<0.05)$. Also, SM1 and pallidum were significantly different $(P<0.05)$. The total SGCE expression level (all isoforms together) was determined in all five control subjects for the various brain regions. Their means did not differ significantly in the different brain regions $(P=0.5)$, suggesting that high exon $11 \mathrm{~b}$ expression is indeed owing to differential isoform expression and not owing to differences in total SGCE expression levels. Overall, $S G C E$ and $S G C E$ exon $11 \mathrm{~b}$ expression levels
Table 3 Expression levels of alternatively spliced SGCE exon $11 \mathrm{~b}$ in human brain regions of two control subjects (454 sequencing)

\begin{tabular}{|c|c|c|}
\hline \multirow[b]{2}{*}{ Region } & \multicolumn{2}{|c|}{ Exon $11 b$} \\
\hline & Subject 1 (\%) & Subject 2 (\%) \\
\hline \multicolumn{3}{|l|}{$H S$} \\
\hline $\mathrm{Hi}$ & 20.84 & 16.59 \\
\hline $\mathrm{Cb}$ & 37.31 & 20.99 \\
\hline NC & 16.89 & 12.48 \\
\hline $\mathrm{Pu}$ & 33.29 & 11.63 \\
\hline GP & 0.68 & 4.09 \\
\hline SN & 5.98 & 12.60 \\
\hline Th & 26.22 & 16.71 \\
\hline $\mathrm{M} 1$ & 21.58 & 25.51 \\
\hline SM1 & 33.92 & 36.79 \\
\hline \multicolumn{3}{|l|}{$R N$} \\
\hline Brain & 73.25 & \\
\hline \multicolumn{3}{|l|}{$M M$} \\
\hline Brain & 49.80 & \\
\hline \multicolumn{3}{|l|}{$Z F$} \\
\hline Embryo & 20.38 & \\
\hline
\end{tabular}

Expression levels of exon $11 \mathrm{~b}$ are given as percentage of all analyzed sequence reads obtained by 454 sequencing.

Abbreviations: HS, Homo sapiens; RN, Rattus norvegicus; MM, Mus musculus;

$\mathrm{Hi}$, hippocampus; $\mathrm{Cb}$, cerebellum; NC, caudate nucleus; Pu, putamen; GP, globus pallidus;

$\mathrm{SN}$, substantia nigra; Th, thalamus; M1, primary motor cortex; SM1, primary somatosensory cortex.

varied among individuals. Normalization with reference genes GAPDH or $e E F-1$ showed comparable results. Furthermore, the 454 sequencing and qPCR results are in support of each other (Supplementary Figure 2).

\section{Localization of SGCE mRNA isoforms in human brain}

To study SGCE mRNA localization of the major brain-specific and the ubiquitously expressed transcripts, we performed isoform-specific ISH in human brain (Figure 3, Supplementary Figure 3). SGCE exon $11 \mathrm{~b}$ and total SGCE expression was most prominent in neurons in all regions tested: high signal in the cerebral cortex, cerebellum (Purkinje cells, dentate nucleus), hippocampus (pyramidal neurons of all CA regions) and moderate signal in the mesencephalon (substantia nigra) and basal ganglia. There was only a faint signal in glial cells and no staining in the white matter. The exon $11 \mathrm{~b}$ probe did not detect a signal in skeletal muscle, heart, kidney and liver, in contrast to the $S G C E$ probe targeting all isoforms. Probe specificity was confirmed with brain tissue of an SGCE null mutation carrier (c.835_839del, p.Lys280SerfsX16) showing no staining for both probes, whereas it stained for control genes (CD68, TSEN54).

In view of the localized expression of $S G C E$ in the cerebellum, we tested whether the interindividual variation of SGCE exon $11 \mathrm{~b}$ expression in the cerebellum is owing to variation in Purkinje cell number in the tissue examined. qPCR analysis of Purkinje cell marker Calbindin-D28k showed no correlation with SGCE expression (data not shown).

\section{Mutation analysis of novel SGCE exons}

We performed mutation analysis in our cohort of definite $\mathrm{M}-\mathrm{D}$ patients where no mutation was identified ${ }^{7}$ and sequenced novel alternatively spliced SGCE exons. This included all new in-frame exons $(1 \mathrm{c}, 4 \mathrm{a})$ and frameshift exon $11 \mathrm{~b}$ with all extended versions 

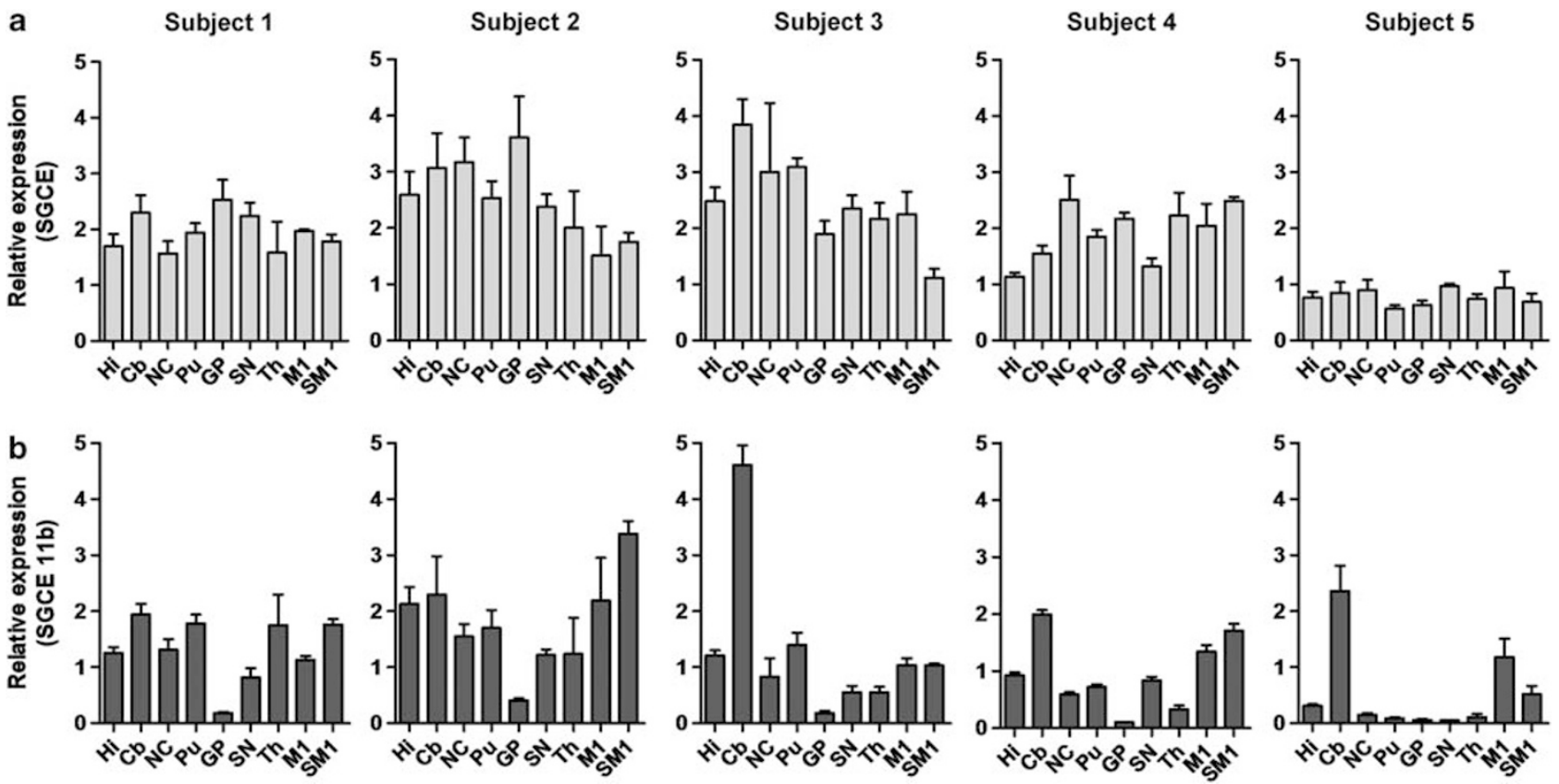

Figure 2 SGCE expression levels (qPCR). Displayed are expression levels for total SGCE (a) and SGCE exon 11b (b) transcripts of the five tested control subjects normalized to GAPDH. Y axis shows SGCE or SGCE11b/GAPDH*100. $\mathrm{Hi}$, hippocampus; $\mathrm{Cb}$, cerebellum; NC, caudate nucleus; $\mathrm{Pu}$, putamen; GP, globus pallidus; SN, substantia nigra; Th, thalamus; M1, primary motor cortex; SM1, primary somatosensory cortex.

(exons 11d, 11c and 11f). Also, frameshift exons $3 \mathrm{~b}$ and $3 \mathrm{~d}$ were sequenced as their combination was observed in a transcript, and maintains the reading frame. No mutations were identified in the 12 patients analyzed.

\section{DISCUSSION}

Mutations in SGCE lead to $\mathrm{M}-\mathrm{D}$, but how mutations in this ubiquitously expressed gene result only in neurological impairments is unknown. In this study, we propose a link between the cerebellum and the M-D phenotype.

$\mathrm{M}-\mathrm{D}$ is an autosomal-dominant disease with a reduced penetrance owing to maternal imprinting. Monoallelic paternal SGCE expression has been shown in human blood leukocytes and in human and mice brain. ${ }^{15,18}$ Several genes are known to show tissue-specific imprinting $^{22}$ and brain cell-type- or region-specific imprinting. ${ }^{23}$ The latter has not yet been investigated for SGCE, but is important to address, as only regions in which the imprint is maintained can be involved in the $\mathrm{M}-\mathrm{D}$ pathogenesis. In this study, we show that the SGCE imprinting pattern is retained in different human brain regions and different human tissues (muscle, blood) similar to what has been shown in mice. ${ }^{18}$ Thus, the brain-specific M-D phenotype cannot be explained by a brain region- or tissue-specific imprinting. As not all $\mathrm{M}-\mathrm{D}$ patients have mutations in SGCE, silencing of SGCE expression owing to imprinting defects could also be a possible disease mechanism. We tested our cohort of patients in which no mutation was identified and found a normal imprint in blood. Imprinting defects in the brain cannot be excluded, however.

More likely, explanations for the brain-specific symptoms in M-D are the presence of a brain-specific SGCE isoform with a brain-specific function or a brain-specific function for ubiquitous SGCE. Our analysis of SGCE expression in human brain using ultra-deep amplicon sequencing shows the presence of 23 alternatively spliced exons, of which 19 at very low frequencies (Table 2). Five of the 23 events retained an open reading frame and are expected to be translated (exons 1c, 2, 4a, 8 and 10). Exons 1c and 4a have not been identified thus far. The majority of the new splice variants have splice sites implying involvement of the splicing machinery. However, most of the new isoforms lead to a frameshift and a premature stop codon, which makes them likely a target for nonsense-mediated decay. Despite the overall low frequency of all new exons, we cannot exclude that they contribute to the physiological function of SGCE.

Of the four major alternatively spliced exons (exon 1c, 2, 8 and $11 \mathrm{~b}$ ), exon $11 \mathrm{~b}$ was of most interest with respect to the disease: it has a brain-specific expression pattern, was highly conserved and expressed at high levels. Exon 8 expression was low in the brain $(7.2 \%)$ compared with heart and muscle (96\%), but its expression in the brain appears to be variable. We further analyzed the expression of $S G C E$ exon $11 \mathrm{~b}$ throughout the human brain. We identified differential expression of exon $11 \mathrm{~b}$ in human brain areas, whereas total SGCE expression levels did not differ. qPCR analysis confirmed this observation and revealed trends: a consistently high expression in the cerebellum, moderate to high expression in SM1, moderate expression in substantia nigra and putamen and consistently low levels in globus pallidus. We observed a high interindividual variability of exon $11 \mathrm{~b}$ expression in the cerebellum. Interindividual differences in the expression of isoforms could account for this observation..$^{24,25}$

We show that exon $11 \mathrm{~b}$ is the major brain-specific alternatively spliced SGCE exon and that it is differentially expressed in five control subjects. Its expression pattern is compatible with a role for the brainspecific $S G C E$ isoform in the cerebellum and a link to M-D. This hypothesis is supported by recent literature: (1) there is a shift from dysfunction of the basal ganglia towards involvement of the cerebellum in dystonia pathogenesis; ${ }^{26}$ (2) several animal models linked dystonia to abnormal cerebellar signaling and cerebellar defects, and cerebellar lesions leading to dystonia have been observed in patients; ${ }^{26,27}$ (3) fMRI analysis of genetically confirmed M-D patients suggests involvement of thalamus and cerebellum (dentate nucleus) ${ }^{28}$ and involvement of different cortical areas and cerebellum. ${ }^{29}$ 


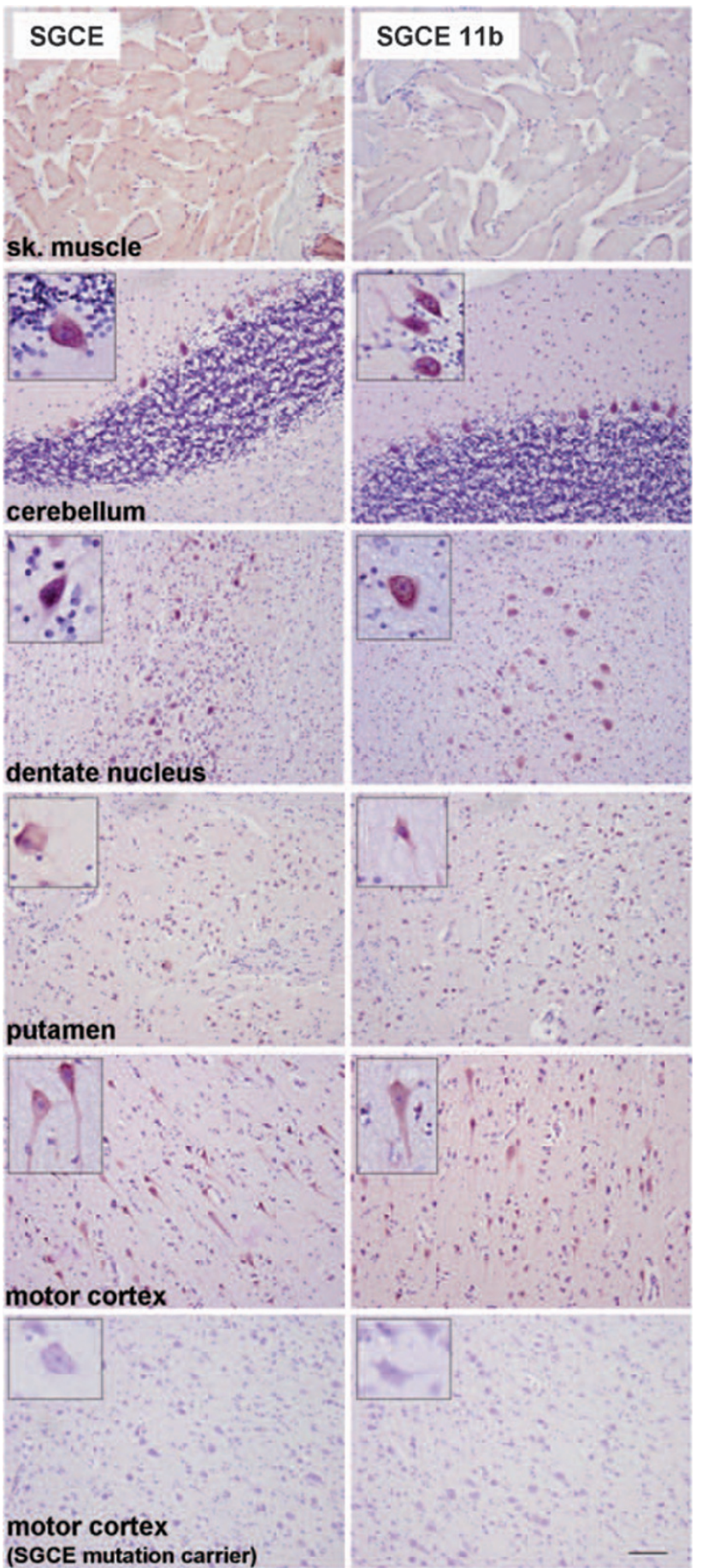

Figure 3 Localization of total SGCE transcripts and those containing exon $11 \mathrm{~b}$. Depicted are ISH results for total SGCE and brain-specific SGCE in different human brain regions and human skeletal muscle. SGCE showed neuronal staining in all brain regions tested as well as staining in the muscle. Brain-specific SGCE transcripts containing exon 11b showed the same expression pattern in the brain, but were not expressed in the skeletal muscle. No SGCE mRNA was detected in the motor cortex of an M-D patient carrying an SGCE nonsense mutation. Insets in each panel show high magnification of neurons. Scale bar represents $100 \mu \mathrm{m}$.

A role of the cerebellum in $\mathrm{M}-\mathrm{D}$ is in contrast with striatal changes on dopamine levels in SGCE-deficient mice and a reduced striatal $\mathrm{D} 2$ receptor binding in $\mathrm{M}-\mathrm{D}$ patients. ${ }^{30,31}$ Moderate expression levels of the major brain-specific SGCE isoform in the striatum and lowest expression levels in the globus pallidus were found in all control subjects. We propose that observed striatal changes may be secondary owing to abnormal cerebellar signaling. This is supported by an anatomical link between the cerebellum and the basal ganglia, namely projections from the dentate nucleus to the striatum, ${ }^{32}$ and by cerebellar lesions or stimulations that have been shown to alter striatal dopamine signaling. ${ }^{33-35}$ Our isoform-specific ISH showed that the major brain-specific SGCE transcript is highly expressed in Purkinje cells and neurons of the dentate nucleus in the cerebellum. No signals were detected in the cerebellar granule cell layer in the cerebellum as it has been shown in mouse brain. ${ }^{17}$ Interestingly, alcohol consumption alleviates the symptoms in $\mathrm{M}-\mathrm{D}$ and the cerebellum reacts very sensitively to alcohol. ${ }^{36}$ Also, symptoms of patients with essential tremor, a common neurological movement disorder with suggested involvement of the cerebellum, ${ }^{37-39}$ are known to respond to alcohol as seen in $\mathrm{M}-\mathrm{D}$.

We propose that the loss of function of the brain-specific SGCE isoform underlies the exclusively neurological M-D phenotype. We hypothesize that the general function of the abundant SGCE isoform is redundant or not essential as no non-neurological symptoms have been reported. The brain-specific protein may have a unique function and cannot be replaced.

\section{CONFLICT OF INTEREST}

The authors declare no conflict of interest.

\section{ACKNOWLEDGEMENTS}

We thank Edward J Bradley for assistance with 454 sequencing and Dr Kees Fluiter for help with ISHs. We thank Dr Anneloor ten Asbroek for helpful discussion and critical reading of the manuscript. Tissue from the $M-D$ mutation carrier was obtained from the Brain and Tissue Bank for Developmental Disorders at the University of Maryland under contracts N01-HD-43368 and N01-HD-4-3383. This study was supported by Nederlandse Organisatie voor Wetenschappelijk Onderzoek (NWO) VIDI (Project No. 0160.056.333) and by the Prinses Beatrix Fund.

1 Klein C: Myoclonus and myoclonus-dystonias; in Pulst SM (ed): Genetics of Movement Disorders. San Diego, CA, USA: Academic Press/Elsevier Science, 2003, pp 449-469.

2 Nardocci N, Zorzi G, Barzaghi C et al: Myoclonus-dystonia syndrome: clinical presentation, disease course, and genetic features in 11 families. Mov Disord 2008; 23: 28-34.

3 Marelli C, Canafoglia L, Zibordi F et al: A Neurophysiological Study of myoclonus in patients with DYT11 myoclonus-dystonia syndrome. Mov Disord 2008; 23 : 2041-2048.

4 Breakefield XO, Blood AJ, Li Y, Hallett M, Hanson PI, Standaert DG: The pathophysiological basis of dystonias. Nat Rev Neurosci 2008; 9: 222-234.

5 Zimprich A, Grabowski M, Asmus $F$ et al: Mutations in the gene encoding epsilonsarcoglycan cause myoclonus-dystonia syndrome. Nat Genet 2001; 29: 66-69.

6 Grunewald A, Djarmati A, Lohmann-Hedrich $\mathrm{K}$ et al: Myoclonus-dystonia: significance of large SGCE deletions. Hum Mutat 2008; 29: 331-332.

7 Ritz K, Gerrits MCF, Foncke EMJ et al: Myoclonus-dystonia: clinical and genetic evaluation of a large cohort. J Neurol Neurosurg Psychiatr 2009; 80: 653-658.

8 Grimes DA, Han F, Lang AE, George-Hyssop P, Racacho L, Bulman DE: A novel locus for inherited myoclonus-dystonia on 18p11. Neurology 2002; 59: 1183-1186.

9 Han F, Racacho L, Lang AE, Bulman DE, Grimes DA: Refinement of the DYT15 locus in myoclonus dystonia. Mov Disord 2007; 22: 888-892.

10 Ozawa E, Mizuno Y, Hagiwara Y, Sasaoka T, Yoshida M: Molecular and cell biology of the sarcoglycan complex. Muscle Nerve 2005; 32: 563-576.

11 Waite A, Tinsley CL, Locke M, Blake DJ: The neurobiology of the dystrophin-associated glycoprotein complex. Ann Med 2009; 41: 344-359.

12 McNally EM, Ly CT, Kunkel LM: Human epsilon-sarcoglycan is highly related to alphasarcoglycan (adhalin), the limb girdle muscular dystrophy 2D gene. FEBS Lett 1998; 422: 27-32.

13 Hjermind LE, Vissing J, Asmus $\mathrm{F}$ et al: No muscle involvement in myoclonus-dystonia caused by epsilon-sarcoglycan gene mutations. Eur J Neurol 2008; 15: 525-529. 
14 Muller B, Hedrich K, Kock $\mathrm{N}$ et al: Evidence that paternal expression of the epsilonsarcoglycan gene accounts for reduced penetrance in myoclonus-dystonia. Am J Hum Genet 2002; 71: 1303-1311.

15 Grabowski M, Zimprich A, Lorenz-Depiereux B et al: The epsilon-sarcoglycan gene (SGCE), mutated in myoclonus-dystonia syndrome, is maternally imprinted. Eur J Hum Genet 2003; 11: 138-144.

16 Esapa CT, Waite A, Locke M et al: SGCE missense mutations that cause myoclonusdystonia syndrome impair epsilon-sarcoglycan trafficking to the plasma membrane: modulation by ubiquitination and torsinA. Hum Mol Genet 2007; 16: 327-342.

17 Nishiyama A, Endo T, Takeda S, Imamura M: Identification and characterization of epsilon-sarcoglycans in the central nervous system. Mol Brain Res 2004; 125: 1-12.

18 Yokoi F, Dang MT, Mitsui S, Li Y: Exclusive paternal expression and novel alternatively spliced variants of epsilon-sarcoglycan mRNA in mouse brain. FEBS Lett 2005; 579: 4822-4828.

19 Ruijter JM, Ramakers C, Hoogaars WM et al: Amplification efficiency: linking baseline and bias in the analysis of quantitative PCR data. Nucleic Acids Res 2009; 37: e45.

20 Budde BS, Namavar Y, Barth PG et al: tRNA splicing endonuclease mutations cause pontocerebellar hypoplasia. Nat Genet 2008; 40: 1113-1118.

21 Maquat LE: Nonsense-mediated mRNA decay: splicing, translation and mRNP dynamics. Nat Rev Mol Cell Biol 2004; 5: 89-99.

22 Albrecht $U$, Sutcliffe JS, Cattanach BM et al: Imprinted expression of the murine Angelman syndrome gene, Ube3a, in hippocampal and Purkinje neurons. Nat Genet 1997; 17: 75-78.

23 Yamasaki K, Joh K, Ohta $\mathrm{T}$ et al: Neurons but not glial cells show reciprocal imprinting of sense and antisense transcripts of Ube3a. Hum Mol Genet 2003; 12: 837-847.

24 Wang ET, Sandberg R, Luo SJ et al: Alternative isoform regulation in human tissue transcriptomes. Nature 2008; 456: 470-476.

$25 \mathrm{Kwan} \mathrm{T}$, Benovoy D, Dias $\mathrm{C}$ et al: Genome-wide analysis of transcript isoform variation in humans. Nat Genet 2008; 40: 225-231.

26 Jinnah $\mathrm{HA}$, Hess EJ: A new twist on the anatomy of dystonia: the basal ganglia and the cerebellum? Neurology 2006; 67: 1740-1741.
27 Pizoli CE, Jinnah HA, Billingsley ML, Hess EJ: Abnormal cerebellar signaling induces dystonia in mice. J Neurosci 2002; 22: 7825-7833.

28 Nitschke MF, Erdmann C, Trillenberg $P$ et al: Functional MRI reveals activation of a subcortical network in a 5-year-old girl with genetically confirmed myoclonus-dystonia. Neuropediatrics 2006; 37: 79-82.

29 Beukers RJ, Foncke EM, van der Meer JN et al: Disorganized sensorimotor integration in mutation-positive myoclonus-dystonia: a functional magnetic resonance imaging study. Arch Neurol 2010; 67: 469-474.

30 Yokoi F, Dang MT, Li J, Li Y: Myoclonus, motor deficits, alterations in emotional responses and monoamine metabolism in epsilon-sarcoglycan deficient mice. J Biochem 2006; 140: 141-146.

31 Beukers RJ, Booij J, Weisscher N, Zijlstra F, van Amelsvoort TA, Tijssen MA: Reduced striatal D2 receptor binding in myoclonu-dystonia. Eur J Nucl Med Mol Imaging 2009; 36: 269-274.

32 Hoshi E, Tremblay L, Feger J, Carras PL, Strick PL: The cerebellum communicates with the basal ganglia. Nat Neurosci 2005; 8: 1491-1493.

33 Nieoullon A, Cheramy A, Glowinski J: Release of dopamine in both caudate nuclei and both substantia nigrae in response to unilateral stimulation of cerebellar nuclei in the cat. Brain Res 1978; 148: 143-152.

34 Tranchant C, Maquet J, Eber AM, Dietemann JL, Franck P, Warter JM: Cerebellar cavernous angioma, cervical dystonia and crossed cortical diaschisis. Rev Neurol 1991; 147: 599-602.

35 Neychev VK, Fan X, Mitev VI, Hess EJ, Jinnah HA: The basal ganglia and cerebellum interact in the expression of dystonic movement. Brain 2008; 131: 2499-2509.

36 Volkow ND, Mullani N, Gould L et al: Effects of acute alcohol intoxication on cerebral blood flow measured with PET. Psychiatry Res 1988; 24: 201-209.

37 Dupuis MJ, Delwaide PJ, Boucquey D, Gonsette RE: Homolateral disappearance of essential tremor after cerebellar stroke. Mov Disord 1989; 4: 183-187.

38 Louis ED, Vonsattel JP, Honig LS et al: Essential tremor associated with pathologic changes in the cerebellum. Arch Neurol 2006; 63: 1189-1193.

39 Louis ED: Essential tremor: evolving clinicopathological concepts in an era of intensive post-mortem enquiry. Lancet Neurol 2010; 9: 613-622.

Supplementary Information accompanies the paper on European Journal of Human Genetics website (http://www.nature.com/ejhg) 